\title{
Universality in Glass Transitions
}

\author{
Roumen Tsekov and Tony Spassov
}

Faculty of Chemistry and Pharmacy, University of Sofia, 1164 Sofia, Bulgaria

\begin{abstract}
The Vogel-Fulcher-Tammann equation is exposed as a particular example of the mean field theory. It is generalized by taking into account an arbitrary critical exponent of susceptibility, discriminating between different classes of universality. The Bell-Evans-Polanyi principle is employed to estimate the difference between the activation energies of flows in crystals and glasses, which appears to coincide with the excess Gibbs energy of the glass compared to the crystal.
\end{abstract}

Keywords: glass transitions, universality, Bell-Evans-Polanyi principle

It is known that the dynamic viscosity $\eta$ of many complex liquids is well described by the empirical Vogel-Fulcher-Tammann (VFT) equation ${ }^{1}$

$\ln \left(\eta / \eta_{0}\right)=B /\left(T-T_{0}\right)$

where $\eta_{0}, B$ and $T_{0}$ are specific constants. When temperature $T$ approaches $T_{0}$, the viscosity increases dramatically, thus leading to glass formation. On the other hand, the temperature dependence of the viscosity defines the activation energy $E_{A}=R T \ln \left(\eta / \eta_{0}\right)$ via the rigorous $A r-$ rhenius law. Introducing here Eq. (1) results in temperature dependent activation energy ${ }^{2}$

$E_{A}=R B /\left(1-T_{0} / T\right)=\varepsilon_{A}(1+C)$

where $\varepsilon_{A} \equiv R B$ is the constant activation energy of a single molecule. The flow in condensed matter is a collective phenomenon and that is why the quantity $C \equiv\left(T / T_{0}-1\right)^{-1}$ can be interpreted as the cooperative number. It shows how many additional molecules should move in the 
liquid to absorb the external force per unit molecule. This definition is normalized by the obvious limit $C(T \rightarrow \infty)=0$, since at infinite temperature no correlations between the fluid molecules are present.

According to the theory of critical phenomena, $T_{0}$ appears to be the critical temperature of the viscosity and one can generalize the expression above by introduction of the critical exponent of susceptibility $\gamma$

$C \equiv\left(T / T_{0}-1\right)^{-\gamma}$

The cooperative number is related also to the Kohlrausch exponent, ${ }^{3}$ which can be generalized by using Eq. (3) to

$$
n=\frac{C\left(T_{m}\right)}{C(T)}=\left(\frac{T-T_{0}}{T_{m}-T_{0}}\right)^{\gamma}
$$

where $T_{m}$ is the melting temperature. The VFT equation (1) corresponds in particular to the mean field theory with $\gamma=1$. There are, however, many other possibilities. ${ }^{4}$ For instance, 3D systems can follow the self-avoiding walk $(\gamma=1.1575)$, the Ising model $(\gamma=1.2373)$, the classical $\mathrm{XY}$ model ( $\gamma=1.3177$ ), the Heisenberg model $(\gamma=1.3955)$, the percolation model $(\gamma=1.7933$ ), etc. Thus, one can relate the universality class of the liquid to a macroscopic model for the dynamic viscosity

$$
R T \ln \left(\eta / \eta_{0}\right)=\varepsilon_{A}\left[1+\left(T / T_{0}-1\right)^{-\gamma}\right]
$$

The activation energy $\varepsilon_{A}$ for the viscous flow in glasses is lower than the activation energy $\varepsilon_{K}$ of the viscous flow in crystals, due to the positive enthalpy of melting $\Delta h_{m}$. Following the BellEvans-Polanyi (BEP) principle, ${ }^{5}$ one can write the relation

$$
\varepsilon_{A}=\varepsilon_{K}-\alpha \Delta h_{m}
$$


where $\alpha=0 \div 1$ is the BEP coupling parameter. Since the liquid can flow only at temperature above the melting point $T_{m}$, one expects the height of the barriers of flow in crystals to be proportional also to the heat of melting, i.e. $\varepsilon_{K} \approx \Delta h_{m}$. On the other hand, the activation energy in glasses should be proportional to the critical temperature, $\varepsilon_{A} \approx T_{0} \Delta s_{m}$, where $\Delta s_{m}=\Delta h_{m} / T_{m}$ is the positive entropy of melting. Thus, Eq. (6) acquires the elegant form $T_{0}=(1-\alpha) T_{m}$ and the BEP parameter can be expressed now as

$\alpha=1-T_{0} / T_{m}$

It is amazing that the decrease of the activation energy $\varepsilon_{K}-\varepsilon_{A}=\alpha \Delta h_{m}=\Delta s_{m}\left(T_{m}-T_{0}\right)$ equals exactly to the excess of Gibbs energy in the glass as compared to the corresponding crystal. Since the glass transition temperature $T_{g} \approx T_{0}$ in metals and alloys is usually half of the melting temperature, it follows from Eq. (7) that the BEP parameter is $\alpha \approx 1 / 2$. Interestingly, the cooperative number at the melting point is one for such systems, $C\left(T_{m}\right) \approx 1$. For organic materials, however, it is known that $T_{g} / T_{m} \approx 3 / 4$, which implies $\alpha \approx 1 / 4$ and $C\left(T_{m}\right) \approx 3^{\gamma}$.

1. J. Rault, J. Non-Cryst. Solids 271 (2000) 177

2. J.P. Sethna, Europhys. Lett. 6 (1988) 529

3. J. Rault, J. Non-Cryst. Solids 260 (1999) 164

4. J.M. Yeomans, Statistical Mechanics of Phase Transitions, Clarendon Press, Oxford, 2002

5. R.P. Bell, Proc. R. Soc. London Ser. A 154 (1936) 414

6. M.G. Evans, M. Polanyi, J. Chem. Soc. Faraday Trans. 32 (1936) 1340 